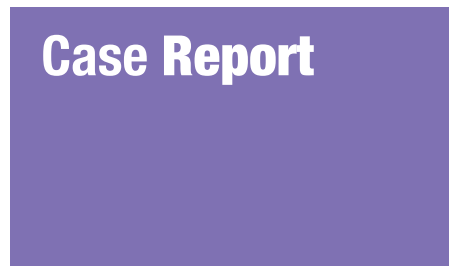

Submitted: 3 Aug 2015

Accepted: 6 Mar 2016

Online: 30 June 2016

\title{
Unicentric Castleman Disease: An Unusual Cause of An Isolated Neck Mass
}

\author{
Anjay Kumar ${ }^{1}$, Krittika AggarwaL ${ }^{1}$, Himanshu AgrawaL ${ }^{1}$, \\ Sonal Sharma ${ }^{2}$, Pankaj Kumar GARG ${ }^{1,3}$
}

1 Department of Surgery, University College of Medical Sciences and Guru Teg Bahadur Hospital, University of Delhi, Delhi, India 110095

2 Department of Pathology, University College of Medical Sciences and Guru Teg Bahadur Hospital, University of Delhi, Delhi, India 110095

3 Department of Surgical Oncology, All India Institute of Medical Sciences, New Delhi, India 110095

To cite this article: Kumar A, Aggarwal K, Agrawal H, Sharma S, Garg PK. Unicentric castleman disease: Unusual cause of isolated neck mass. Malays J Med Sci. 2016; 23(4):86-89. doi: 10.21315/mjms2016.23.4.12

To link to this article: http://dx.doi.org/10.21315/mjms2016.23.4.12

\begin{abstract}
Castleman disease (CD) is a rare lymphoproliferative disorder of unknown aetiology. It manifests in two distinct clinical presentations: unicentric and multicentric. Unicentric CD is rare and may present as an isolated neck mass. A 22-year-old man presented with a 6-month history of right neck swelling that occupied the posterior triangle of the right neck region. After surgical exploration, a solitary, well defined, and hyper vascular mass was excise. A histopathological examination confirmed the lesion as CD, hyaline-vascular variant. CD of the neck is a diagnosis that is usually not taken into consideration while evaluating neck masses due to its rarity and unassuming presentation. It should be keep in the differential diagnosis of neck masses as the clinical and radiological features evade a firm diagnosis. The treatment of unicentric CD is complete surgical excision, which cures the patient.
\end{abstract}

Keywords: Castleman disease; lymphadenopathy; lymphoproliferative disorder

\section{Introduction}

Castleman disease (CD) is a rare lymphoproliferative disorder known by a myriad of other names reflecting the fact that much is still to be discover about the aetiopathogenesis of this disease. CD was first describe by Dr. Benjamin Castleman in 1954 in a patient who had an isolated solitary hyperplastic mediastinal lymph node with a regressive germinal centre. Clinically, two distinct subtypes of CD have been identified-unicentric (localised) and multicentric (systemic) (1). Unicentric CD presents as an enlargement of a single lymph nodal chain or area (most commonly in the mediastinum and other sites being the neck, abdomen and axilla). This form is usually asymptomatic and potentially curable by surgical excision. However, it also rarely presents as an extra-nodal disease. The absence of defining features on imaging makes excision with a histopathological examination mandatory for confirming the diagnosis. The aetiology ranges from viral infection to various unknown disturbances at the B-cell level resulting in a proliferation of $\mathrm{B}$ cells around the atrophic and small germinal centres (in an "onion skin" pattern), which are characteristic of the disease (2). Unicentric CD should be keep in the differential diagnosis of neck masses. However, caution must be exercise in performing an excision biopsy without adequate preparation as the hypervascularity of the lesion may cause an excessive blood loss (2). This disorder remains a diagnostic challenge for many clinicians. 


\section{Case Description}

A 22-year-old man presented with a 6-month history of a progressively enlarging and painless right neck swelling. There was no history of tuberculosis. He was in good health and was worried about the growing mass. A systemic examination was unremarkable. The swelling occupied the posterior triangle of right neck and was of firm consistency with a smooth surface (Figure 1).

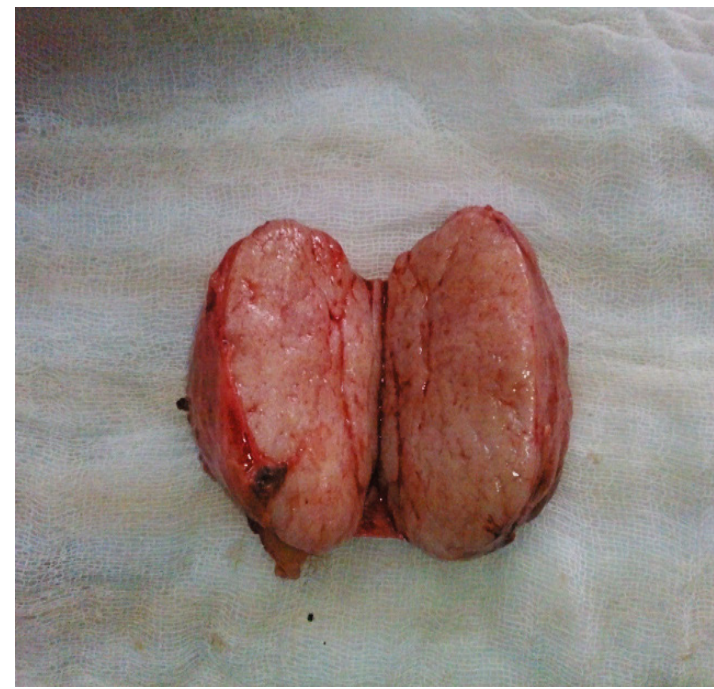

Figure 1: Preoperative photograph of the patient

The carotid artery was not displace. No neurological symptoms or signs were present. Contrast-enhanced computed tomography (CECT) of the neck showed an $8 \times 5 \mathrm{~cm}$ mass adjacent to the right carotid artery with moderate contrast enhancement. No necrotic or ring enhancement was evident. CECT of the thorax and abdomen was unremarkable. The patient was sero-negative for HIV. A fine needle aspiration cytology (FNAC) of the neck mass raised the suspicion of lymphoma. A decision for an excision biopsy under general anaesthesia was made. After surgical exploration, a solitary, well define, hypervascular mass was excised. A cut section of the resected mass showed a well circumscribe, multi-lobulated yellowish tumor (Figure 2).

A microscopic examination showed numerous lymphoid follicles present throughout the lymph node with an involuted germinal centre. Higher magnification revealed a follicle containing arterioles with plump endothelial cells surrounded by an onion-skin layering of lymphocytes (Figure 3); thus, confirming the lesion as a hyaline-vascular variant of $\mathrm{CD}$. The patient was well and asymptomatic after 2 years of follow-up.

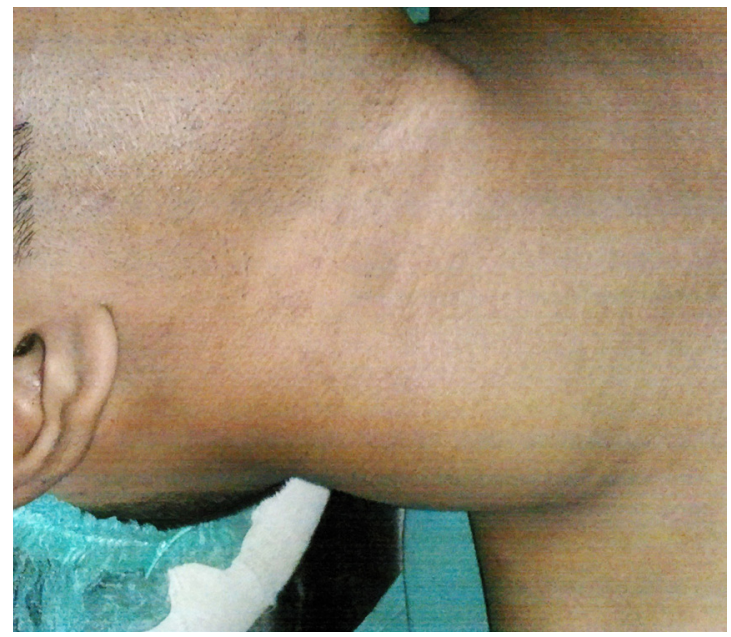

Figure 2: Cut section of the neck mass shows well-circumscribed, multi-lobulated yellowish tumor

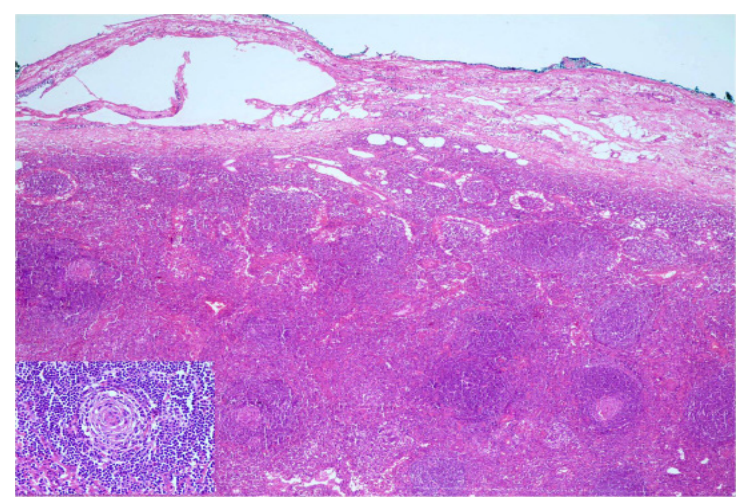

Figure 3: (H\&E x40) photomicrograph shows numerous lymphoid follicles present throughout the lymph node with involuted germinal centres. Inset (H\&E x400) shows follicle containing arteriole with plump endothelial cells surrounded by 'onion-skin' layering of lymphocytes.

\section{Discussion}

$\mathrm{CD}$, a lymphoid tissue disorder, is a rare occurrence with an uncertain aetiopathogenesis. Because of its rarity, it is commonly misdiagnose and mistaken for a number of other aetiologies. Various synonyms by which CD is known are testimony to this fact (3). Though first describe by Benjamin Castleman in 1954 and later histopathologically characterised by Flendrig 
and Keller in the 1970s, the disorder still eludes classification as a disorder, hyperplasia, hamartoma or a response to inflammation or infection (2). Three histological variants are now recognised-hyaline vascular, plasma cell and plasmablastic (1). The hyaline variant is a lymphoid proliferation wherein the follicles are regressed or depleted of germinal centre cells and have expanded mantle zones with small lymphocytes arranged concentrically in an onion-skin fashion. In the plasma cell variant, the lymph nodes reveal a preserved architecture with hyperplastic follicles with increased interfollicular plasma cells being the most striking feature. In human herpes virus-8 (HHV8)-associated multicentric $\mathrm{CD}$, a number of immunoblasts (also called plasmablasts) present in the outer mantle zones of some follicles; this is considered a separate entity known as the plasmablastic variant.

Clinically, CD presents in two forms, unicentric and multicentric. The unicentric form is more common and presents as a solitary mass with a progressive enlargement and indolent course. Approximately 90\% of the unicentric forms are of the hyaline-vascular type. The multicentric or diffuse form is plasma cell variant and it presents with systemic manifestations including fever, fatigue, anaemia, hyperglobulinaemia, an elevated sedimentation rate and diffuse lymphadenopathy.

Unicentric CD occurs with equal frequency in men and women and is commonly seen in the second and third decades of life. The most common site for isolated $\mathrm{CD}$ is the mediastinum (about $70 \%$ of cases). The cervical region is the next most common site accounting for $15 \%-20 \%$ of cases (4). However, CD can occur at any lymph node site and at extranodal sites harbouring lymphoid tissue. The aetiology of $\mathrm{CD}$ remains a matter of conjecture. Excessive production of inteleukin-6 (IL-6) has been demonstrate in CD lesions; this indicates a possible role of viral infections in the causation of CD. Increased IL-6 results in the increased proliferation and survival of B cells, which is the hallmark symptom of CD. Multicentric CD is associated with HHV-8 in HIV-positive individuals; however, the role of viruses in the causation of CD in non-HIVpositive individuals remains to be determined and is a subject of active investigations. CD has also been reported to occur in association with both Hodgkin and non-Hodgkin lymphomas. On the other hand, the rich and typical vascularity of the lesion, the absence of lymphatic sinuses, its extranodal occurrence and the absence of recurrence favours a hamartomatous origin of $\mathrm{CD}$ (5). Others have proposed that $\mathrm{CD}$ is due to an immunological disorder, possibly at the B lymphocyte level. A neoplastic origin for unicentric $\mathrm{CD}$ has been discard due to its rarity of recurrence.

A preoperative diagnosis remains difficult due to its non-diagnostic features on imaging. On CECT of the neck, CD is seen as a homogenous mass lesion with moderate to intense enhancement owing to the vascularity of the lesion; this enhancement is more marked in the hyaline-vascular variant. A ring enhancement of the lesion has also been describe in one case of $\mathrm{CD}$ of the neck. In a study of four patients with $\mathrm{CD}$ of the neck, a central non-enhancing scar in an otherwise enhancing lymph node mass has been purported as an important diagnostic clue (5). In the absence of pathognomic features, the list of differential diagnoses is long and includes lymphoma, tuberculosis, metastatic thyroid carcinoma and infective conditions like cat scratch fever, Epstein-Barr virus infection, HIV, Kaposi sarcoma and Kimura disease. FNAC often shows lymphoid tissue resulting in an erroneous diagnosis of lymphoma, as seen in our case.

Complete surgical excision followed by a histopathological evaluation is required as a mode of confirmation of diagnosis in isolated CD and for its treatment. Preoperative embolisation may reduce blood losses (2); however, it is infrequently employed as the preoperative diagnosis is uncertain in most cases. In the hyaline-vascular variant, recurrences are unknown in isolated $\mathrm{CD}$; however, recurrences have been seen in plasma cell variants. Though radiation therapy is advise in the event of an incomplete surgical excision, the benefit is marginal. A favourable outcome is still expected because of the indolent nature of the disease.

Multicentric CD is difficult to treat and has an unfavourable long-term prognosis. The use of combination chemotherapy with or without steroids has resulted in significant remissions. However, the disease tends to persist from months to years and results in fatal outcomes due to its evolution into neoplasia like Kaposi sarcoma, lymphoma or plasmacytoma, or to infectious complications (1).

\section{Conclusion}

$\mathrm{CD}$, though rare, should be considered in the differential diagnosis of neck masses as it is a potentially curable condition. Complete surgical extirpation of the mass is curative in unicentric 
variant. It still remains an enigmatic disease more than 50 years after its first description and poses an array of questions regarding its aetiopathogenesis and management that will fuel further research to better characterise this entity.

\section{Acknowledgement}

None

\section{Conflict of Interests}

The authors have no conflict of interest to declare.

\section{Funds}

None

\section{Authors Contributions}

Conception and design, data acquisition and analysis, and drafting the article : AK, KA, HA, PKG and SS Managed the patient clinically : AK, KA, HA and PKG Performed the histopathological examination of the specimen : SS

\section{Correspondence}

Dr Pankaj Kumar Garg

MBBS (Aligarh Muslim University), MS (University of Delhi), MCh (New Delhi), UICC fellowship (University of Texas)

Department of Surgery,

University College of Medical Sciences and Guru

Teg Bahadur Hospital,

Dilshad Garden, Delhi, India 110095

Tel: +91 1122692536

Email: dr.pankajgarg@gmail.com

\section{References}

1. Casper C. The aetiology and management of Castleman disease at 50 years: translating pathophysiology to patient care. $\mathrm{Br}$ J Haematol. 2005;129:3-17.

2. Newlon JL, Couch M, Brennan J. Castleman's disease: three case reports and a review of the literature. Ear Nose Throat J. 2007;86:414-418.

3. Chaloupka JC, Castillo M, Hudgins P. Castleman disease in the neck: atypical appearance 133 on CT. AJR Am J Roentgenol. 1990;154:1051-1052.

4. Glazer M, Rao VM, Reiter D, McCue P. Isolated Castleman disease of the neck: MR findings. AJNR Am J Neuroradiol 1995;16:669-671.

5. Tan TY. Castleman's disease of the neck: a description of four cases on contrast-enhanced CT. Br J Radiol 2004;77:253-256. 